\title{
Changing Practices in Home Inodilator Infusion Therapy for Advanced Congestive Heart Failure
}

\author{
Monica Barrington
}

\begin{abstract}
A progressive and ultimately fatal disease, congestive heart failure (CHF) is the primary reason for hospitalization of people aged 65 or older. Because this age group is the most rapidly growing segment of our population, finding effective treatments for CHF that maximize quality of life while minimizing overall costs, especially those associated with protracted hospitalizations, is of great concern. The objective of this paper is to review the current standard of care and associated costs of inodilator infusion therapy in patients with CHF and to assess the potential of home infusion care.

Inodilator therapy has shown great promise in improving quality of life for patients with CHF. Moving patients from an inpatient setting to outpatient care with infusion therapy has resulted in subsequent reductions in admission rates, length of stay, and readmission rates.
\end{abstract}

The next step in decreasing health care costs is to shift services from the outpatient clinic system to home care. With proper training, home health care nurses and pharmacists can provide inodilator therapy in the comfort of the patient's home at a cost that may be significantly less than providing this service in a hospital or clinic setting.

Inodilator therapy in a home care setting would reduce the number of hospitalizations, decrease treatment costs, and improve quality of life for patients who have advanced CHF.

Staff education, policy and protocol preparation, and coordination with physicians and primary caregivers will be essential. Future cost-effectiveness studies with home inodilator therapy should be conducted to demonstrate these advantages.

KEY WORDS: Infusion therapy

J Managed Care Pharmacy 1998; 4: 73-77

\section{Author}

MONICA BARRINGTON, R.PH., M.PH., FA.S.C.P, B.C.PM, is Account Manager, at VHA, Inc., Matthews, NC.

AUTHOR CORRESPONDENCE: Monica Barrington, R.Ph., M.Ph., FA.S.C.P, B.C.PM, VHA, Inc., 521 East Morehead, Suite 380, Matthews, NC 29202.

ACKNOWLEDGMENT: Supported by an unrestricted educational grant from Sanofi Pharmaceuticals, Inc.

Copyright (C 1998, Academy of Managed Care Pharmacy, Inc. All rights reserved.
$\mathrm{T}$ he number of patients with congestive heart failure $(\mathrm{CHF})$, the top-ranked diagnosis-related group, is increasing by $5 \%$ annually. ${ }^{1}$ Reports from the Health Care Financing Administration indicate that in 1992, Medicare paid $\$ 2.4$ billion for 654,000 hospitalizations for $\mathrm{CHF}^{2}$ In the United States more than $\$ 10$ billion is spent annually on the care of these patients. ${ }^{3}$ Patients with this disease present at hospital emergency rooms in crisis 1.6 times per year on average, and $90 \%$ require admission to the hospital, indicating that the management of $\mathrm{CHF}$ is less than ideal. ${ }^{4}$ The cost spiral created increases the burden on health care providers, resources, and insurers. Consequently, effective management of $\mathrm{CHF}$ is of great and immediate concern.

Patients with advanced CHF (New York Heart Association [NYHA] functional class III or class IV CHF) are the most critically ill of cardiac patients and the greatest financial burden to the health care system. Guidelines for the treatment of CHF have been established by the Agency for Health Care Policy and Research, but they do not address the needs of the patient with advanced $\mathrm{CHF}^{5}$ One emerging strategy is the development of regional or local CHF-care delivery systems able to provide a fully integrated care plan that would reduce the need for hospitalization and improve the quality of life for heart-failure patients. This article examines the role of a home inodilator infusion program for advanced $\mathrm{CHF}$ as an essential component of this integrated approach that could reduce hospitalizations and enhance patient quality of life. 


\section{SHIFTING SERVICES}

In the past decade, health care delivery has focused on cost management and efficiency. Limitations have been set on expensive diagnostic procedures for inpatients who have $\mathrm{CHF}$ and on length of hospitalization. Some of the costs associated with advanced CHF are those involved with inodilator infusions, including three to four days of hospitalization as well as drug administration and monitoring of patients. Many hospitals have implemented the use of outpatient clinics for frequent patient contact, medication adjustments, patient education, and inodilator infusions. Studies have documented the cost savings of this shift from inpatient to outpatient infusion therapy, which has been associated with a subsequent reduction in admissions rates, length of stay, and readmission rates. ${ }^{6.7}$

The next step in decreasing health care costs was to shift services from the outpatient clinic system to home care. This shift was thought to be most effective for critically ill patients who had decreased accessibility and mobility. For patients with advanced $\mathrm{CHF}$, home inodilator infusion therapy made sense economically and from a quality-of-life perspective. ${ }^{8}$

\section{INODILATOR INFUSION THERAPY}

The home infusion industry began as an attempt to provide various intravenous therapies to patients outside the hospital and clinic environment to reduce high hospital costs, overall costs of therapy, and disruptions to the patient's life that result from standard in-hospital treatments. The home infusion industry initially was reluctant to include the use of chronic or intermittent intravenous inodilator agents for managing advanced CHF due to the risk of side effects, the potential for higher mortality, and the need for specialized cardiac monitoring. However, the home infusion industry has changed its view because the aging patient population is expanding. Now there is an urgent need to decrease costs, and recent clinical findings have demonstrated the efficacy and utility of inodilator infusions. ${ }^{6.7}$ There is an emerging consensus that quality-of-life considerations may be as or more important than mortality for patients with advanced CHF. In addition, the developing expertise of nurses in home infusion therapy makes expansion of this service more practical. Catecholamines, such as dopamine and dobutamine, are the inotropic agents that have been used the longest. Dobutamine is used more frequently, but a recent report shows a reduced hemodynamic effect over long-term use and increased myocardial oxygen consumption at high doses, possibly worsening CHF. ${ }^{9}$

The newer agents, phosphodiesterase inhibitors, are effective without the tolerance or increased cardiac demands. Milrinone, the most commonly used, has shown a distinct advantage over dobutamine for patients with pulmonary vascular resistance such as that seen in impaired ventricular functioning; it is also less proarrhythmic. ${ }^{10}$ Milrinone also has been effective in status management in cardiac transplant patients. ${ }^{11}$

Milrinone has practical advantages over other agents for home health care. It is chemically stable in solution, and a week's supply can be placed in a small (less than $500 \mathrm{ml}$ ) container, easily carried by most patients. These properties make the option of home inodilator infusion therapy with milrinone feasible and potentially cost effective in the treatment of advanced CHF.

\section{REQUIREMENTS FOR HOME INODILATOR SERVICES}

\section{Patient Population}

The patients that would benefit most from home inodilator therapy are those with advanced CHF requiring continuous or intermittent inodilator therapy and a high hospital readmission rate. Patients awaiting heart transplantation may now do so at home with inodilator therapy and not risk serious deterioration of their condition during the process. Management with inotropic agents has shown a $228 \%$ cost saving over traditional mechanical support for patients awaiting heart transplantation. ${ }^{12}$ Cost savings in this study were based on the calculated cost per additional day of survival and the cost-per-survivor index.

Once referred by a cardiologist, a patient would be required to have the following: a reliable caregiver in the home, a telephone, and a central or long peripheral line for continuous infusion or an acceptable intravenous access for intermittent therapies. In addition, the patient should meet the homebound classification for Medicare reimbursement.

\section{Program Initiation}

Policies on the standards of care of patients must be established. The organization that offers home inodilator services must be able to ensure competent and empathetic care of fragile patients with advanced CHF. Protocols and order sets should be prepared in advance with the physicians' input. Physicians assume the primary responsibility for their patients well-being, and they must feel comfortable with the quality of service provided by the home infusion organization. The competence exhibited by representatives of the home infusion organization is of paramount importance in marketing this service to physicians, who over time become referral sources.

The pharmacist's role varies depending on the structure of the home health care organization. However, pharmacists should be thoroughly versed in the actions and side effects of the medications and the use of various infusion pumps. They should also be able to communicate instructions clearly by telephone to both nurses and caregivers. Visiting nurses should possess strong assessment skills and preferably have a background in critical or cardiac care.

\section{Inodilator Dosing}

Milrinone is effective for various advanced CHF presentations and has a good safety profile. For intermittent therapy, a milrinone loading dose of $50 \mathrm{mcg} / \mathrm{kg}$ is administered over 10 to 20 minutes, followed by a dose of $0.5 \mathrm{mcg} / \mathrm{kg}$ per minute 
over four to six hours. Therapists should advise patients that a noticeable reduction in symptoms and an increase in energy often is experienced after the dose of this medication; however, this reaction is transitory and subsides between treatments. The patient's response indicates whether one, two, or three weekly infusions are needed. If a patient's need exceeds three infusions weekly, continuous therapy should be considered. For continuous therapy, milrinone dosing may begin at 0.25 $\mathrm{mcg} / \mathrm{kg}$ per minute and increase to $0.5 \mathrm{mcg} / \mathrm{kg}$ per minute.

Inodilator agents are mixed in a laminar hood or workbench under aseptic conditions. Milrinone is most stable in a concentrated state; therefore, minimal dilution is recommended. Admixed medication should be placed in containers spiked in a clean environment and appropriate for the volumetric pump used. Ambulatory pumps are best for continuous infusion, but accurate pole-mounted pumps may be used for intermittent dosing.

\section{Monitoring}

Because significant hypotensive effects may occur with the concurrent administration of an inodilator agent and some antihypertensive medications, the home health nurse should obtain a thorough medication history before the first scheduled visit so that a decision can be made about appropriately administering antihypertensive medications on inodilator therapy days. The assessment skills of the home health nurse must be keen. Although the incidence of ventricular arrhythmias associated with the use of milrinone is low, patients receiving this medication should be carefully monitored. Furthermore, it should be noted that a $15 \%$ to $20 \%$ reduction in systolic blood pressure during infusion warrants a $50 \%$ reduction in the dose infused or even discontinuation of the infusion. Tables 1 and 2 present subjective and objective monitoring aids for drugrelated problems associated with inotropic therapy. ${ }^{13}$

Because many patients receiving inodilator medications also require diuretic therapy, at least monthly laboratory specimens should be drawn to allow evaluation of electrolyte imbalances. The nurse also should evaluate symptomatology associated with cardiac output, gas exchanges, and cerebral perfusion during the assessment. Finally, the patient should receive monthly follow-up, either in the physician's office or in a clinic.

\section{OUTCOMES}

\section{Quality of Life}

Improved quality of life is becoming an important endpoint in treating advanced CHF. Quality of life is defined by

Table 1. Subjective Monitoring for Drug-Related Problems of Milrinone Therapy ${ }^{11}$

\begin{tabular}{|c|c|c|c|}
\hline Problem & Indicator & Collected by & Options \\
\hline Arrhythmia & $\begin{array}{l}\text { Cardiac awareness; skipping } \\
\text { or rapid heart beat; episodic } \\
\text { shortness of breath or difficulty } \\
\text { in breathing, dizziness or light- } \\
\text { headedness, or syncope }\end{array}$ & Patient or caregiver & $\begin{array}{l}\text { - Provide immediate physician } \\
\text { attention } \\
\text { - Obtain EKG } \\
\text { - Draw blood: treat hypokalemia, } \\
\text { hypomagnesemia } \\
\text { - Decrease dose } \\
\text { - Discontinue drug } \\
\text { - Usually do not treat with } \\
\text { antiarrhythmic drug }\end{array}$ \\
\hline $\begin{array}{l}\text { Hypotension (amrinone, } \\
\text { milrinone) }\end{array}$ & Dizziness or feeling faint & Patient or caregiver & $\begin{array}{l}\text { - Provide immediate physican } \\
\text { attention } \\
\text { - Blood pressure standing and } \\
\text { sitting } \\
\text { - Rule out arrhythmia }\end{array}$ \\
\hline Angina & $\begin{array}{l}\text { Chest pain (patients with } \\
\text { ischemic heart disease) }\end{array}$ & Patient, caregiver, or clinician & $\begin{array}{l}\text { - Provide physician attention } \\
\text { - Give nitroglycerin } \\
\text { - Obtain EKG } \\
\text { - Decrease dose } \\
\text { - Discontinue drug }\end{array}$ \\
\hline Headache & $\begin{array}{l}\text { Headache, especially if associated } \\
\text { with dose increase }\end{array}$ & Patient or caregiver & $\begin{array}{l}\text { - Continue to monitor } \\
\text { - Decrease dose } \\
\text { - Treat with acetaminophen }\end{array}$ \\
\hline Nausea & $\begin{array}{l}\text { Nausea, especially if associated } \\
\text { with dose increase }\end{array}$ & Patient or caregiver & $\begin{array}{l}\text { - Continue to monitor } \\
\text { - Decrease dose } \\
\text { - Treat with antiemetic }\end{array}$ \\
\hline $\begin{array}{l}\text { Thrombocytopenia } \\
\text { (amrinone) }\end{array}$ & $\begin{array}{l}\text { Bruising, bleeding from gums, } \\
\text { black stools }\end{array}$ & Patient or caregiver & $\begin{array}{l}\text { - Provide immediate physican } \\
\text { - Drtention } \\
\text { - Hold amrinone }\end{array}$ \\
\hline
\end{tabular}


Table 2. Objective Monitoring for Drug-Related Problems of Inodilator Therapy ${ }^{11}$

\begin{tabular}{|c|c|c|c|}
\hline Problem & Indicator & Collected by & Options \\
\hline Hypotension & $\begin{array}{l}\text { Reduction diastolic }>20 \mathrm{~mm} \mathrm{Hg} \text {, } \\
\text { especiallywith dose changes }\end{array}$ & $\begin{array}{l}\text { Patient/caregiver daily, } \\
\text { or home care nurse on } \\
\text { visits }\end{array}$ & $\begin{array}{l}\text { - Provide immediate } \\
\text { physician attention } \\
\text { - Decrease dose } \\
\text { - Discontinue drug }\end{array}$ \\
\hline Bradycardia & Reduction >15 bpm & $\begin{array}{l}\text { Patient/caregiver daily, } \\
\text { or home care nurse on } \\
\text { visits }\end{array}$ & $\begin{array}{l}\text { - Provide immediate } \\
\text { physician attention } \\
\text { - Decrease dose } \\
\text { - Discontinue drug }\end{array}$ \\
\hline Arrhythmia & $\begin{array}{l}\text { Pulse irregularity; auscultation } \\
\text { of apical pulse; orthostatic change } \\
\text { (rule out dehydration); sudden } \\
\text { worsening of CHF (rales, edema) }\end{array}$ & Nurse & $\begin{array}{l}\text { - Provide immediate } \\
\text { physician attention } \\
\text { - Obtain EKG } \\
\text { - Draw blood; treat } \\
\text { hypokalemia, hypo- } \\
\text { magnesemia } \\
\text { - Decrease dose } \\
\text { - Discontinue drug }\end{array}$ \\
\hline Increased risk of arrhythmia & $\begin{array}{l}\text { Serum potassium }<3.5 \mathrm{mg} / \mathrm{dL} \\
\text { Serum magnesium }<2.0 \mathrm{mg} / \mathrm{dL}\end{array}$ & Lab draw & $\begin{array}{l}\text { - Treat hypokalemia, } \\
\text { hypomagnesemia }\end{array}$ \\
\hline
\end{tabular}

myriad variables, but the patient's functional capacity, symptom level, and psychosocial adjustment are critical components. ${ }^{14.15}$ Quality of life for patients who have advanced CHF often is compromised by the activity-limiting nature of the disease. Disruptions caused by frequent hospitalizations or outpatient visits to the hospital or clinic can further reduce many patients' perception of life quality. With home health therapy, patients experience less disruption. Home health care also reinforces the patients' entire therapeutic regimen, enhancing compliance and the chance for successful symptom control.

In two separate studies, responses given by participants in outpatient infusion clinics on the Minnesota Living With Heart Failure questionnaire revealed improvements in quality of life after initiation of inodilator therapy. ${ }^{16,17}$ Other studies ${ }^{7}$ also found that quality of life appears to be enhanced directly by the improved functioning and exercise tolerance provided by intravenous inodilator therapy, although they do not report how they measured quality of life. Some of the patients who were once unable to leave their hospital beds unattended reported that after undergoing inodilator therapy they could walk to the mailbox, attend weekly club meetings, resume sexual activities, and take part in other daily activities. Such remarkable results, although not routine, underscore the value of intravenous inodilator therapy and its potentially critical role in home therapy.

\section{Economic Effects}

The use of inodilator medications in outpatient therapy has resulted in reduced hospital and overall health care costs. A recent study of 36 patients with advanced CHF demonstrated that the infusion of inotropic agents in an outpatient clinic program can have a dramatic effect on the cost of care. ${ }^{18}$ Patients who have advanced CHF who participated in this program had statistically significant reductions in their hospital admissions ( 66 to 17), emergency room visits (16 to 2 ), and inpatient hospital days (424 to 58) during an evaluation period that averaged 294 days. These reductions resulted in an $86 \%(\mathrm{p}<0.001)$ decrease in expenditures. Similar savings are anticipated with home infusion programs. The savings with the use of inotropic agents result primarily through a reduction in inpatient hospital stays and emergency room visits. These savings alone could justify a hospital's decision to use home infusion as an alternative to both inpatient and outpatient clinic therapy for managing advanced CHF. This economic benefit may provide a valuable marketing tool for the home infusion provider.

Reimbursement from Medicare is now available for home inodilator therapy through some regional carriers when appropriate diagnostic measures, including pulmonary capillary wedge pressure and cardiac index, support the medical necessity and efficacy of this therapy. The potential cost effectiveness of home inodilator therapy has convinced most private and capitated payors to reimburse for this therapy.

\section{DISCUSSION}

Staff members of a traditional home infusion program may lack the expertise specific for inodilator therapies. External education programs as well as the development of competencies internally should be undertaken to ensure that all home health care nurses involved in this program can provide the care needed by patients who have advanced CHF. 
In addition to possible educational expenses, the purchase of medication and supplies as well as the time required of the nursing and pharmacy staff can increase costs considerably. Because reimbursement margins for this procedure are low, it is important to minimize the costs for equipment, supplies, and staff (nursing and pharmacy) time without jeopardizing the quality of care provided to the patient. Staff time can be saved by the use of telephone assessments, which can easily monitor trends in the patient's condition and reinforce the patient's com pliance. A phone assessment log sheet that includes questions about weight, number of angina episodes, sleep disturbances or patterns, diet, and medication compliance can be useful for the patient, caregiver, and home health care nurse and can take as little as five minutes to complete.

Another obstacle facing the widespread implementation of the home inodilator program is physician reluctance. Physicians who opt for home-bound care incur a reduction in income: fewer inpatients visited daily means reduced reimbursement. Even though reimbursement for managing a patient at home is minimal, the physician is still required to complete paperwork and respond to phone calls. Thus, in many ways, the time demand would be equivalent to caring for an inpatient. To minimize demand on the physician's time, home health care providers can create and provide clear and complete routine order sets that can reduce phone inquiries to the physician and also provide the documentation needed to establish medical necessity, a requirement for insurance provider reimbursement. In light of potential cost savings, physicians are being challenged to change treatment philosophies.

A team approach can be used to overcome most of the obstacles. Collectively, home health care providers, physcians, and caregivers can work toward a single goal: improving the health and well-being of the patients in their care who have advanced $\mathrm{CHF}$

\section{CONCLUSION}

With outpatient intravenous inodilator therapy, patients who have advanced CHF experience an increase in functional status and a decrease in hospitalizations, and those who require hospitalization have a shorter stay. Home infusion therapy programs that incorporate intravenous inodilator agents are expected to provide many of the same benefits. These benefits not only are potentially cost effective, but they also may enhance patients' quality of life. Home inodilator therapy with agents such as milrinone potentially offers clear clinical and economic advantages that make this an attractive alternative for treating advanced CHF.

To reach these goals, education of staff, preparation of policies and protocols, and development of order sets and clinical paths in coordination with the physician and primary caregivers will prove essential. Future cost-effectiveness studies of home inodilator therapy are encouraged to demonstrate the potential benefits of this treatment.
References

1. Health Care Investment Analysts Inc., Ernst \& Young LLP. Medicare summary information for top five diagnosis-related groups. Baltimore, MD: Health Care Investment Analysts Inc. and Ernst \& Young LLP, 1995.

2. Health Care Financing Administration (HCFA). MedPAR database. Baltimore, MD: HCFA Bureau of Data Management and Strategy, Office of Statistics and Data Management, 1994.

3. American Heart Association. Heart and stroke facts: 1994 statistical supplement. Dallas, TX: American Heart Association National Center, 1993 4. Field JL, Van Hoose M. Beyond four walls: cost effective management of chronic congestive heart failure. Washington, DC: The Advisory Board Company, 1994.

5. Heart failure: management of patients with left-ventricular systolic dysfunction. Quick Reference Guide for Clinicians No. 11; Rockville, MD: Agency for Health Care Policy and Research, Public Health Service, US Dept of Health and Human Services; June 1994. AHCPR Publication No. 94-0613. 6. Friedman AW, Minella RA, Silver SJ, et al. Reduced hospital admissions with chronic inotropic therapy for resistant congestive heart failure. CHF 1995(Nov/Dec); 19-23.

7. Jennison SH, Dhar SC, Derfler MC, Miller, LW. Outpatient use of continuous intravenous milrinone for advanced heart failure. CHF 1996(Sep/Oct); $15-20$.

8. Boger JE, DeLuca SL, Watkins DF, et al. Infusion therapy with milrinone in the home care setting for patients who have advanced heart failure. J of Intravenous Nursing 1997; 20: 148-154.

9. Shipley JB, Tolman D, Hastillo A, Hess ML. Milrinone: basic and clinical pharmacology and acute and chronic management. Am J Med Sci 1996; 311(6): 286-291.

10. Marius-Nunez AL, Heaney L, Silber E. The role of intermittent inotropic therapy in the heart failure clinic of Michael Reese Hospital and Medical Center. CHF 1995(Jul/Aug); 30-33.

11. Canver CC, Cooler SD. The efficacy of intravenous milrinone in status I cardiac transplant patients. Paper presented at the American Society of Transplant Physicians 15th Annual Meeting. Dallas, TX: 1996 May 26-30.

12. Loisance D, Sailly JC. Cost-effectiveness in patients awaiting transplantation receiving intravenous inotropic support. Eur J Anesthersiol 1993; 10(sup.8): 9-13.

13. Carroll T. Home care of severe heart failure with intravenous diuretics and inotropes. Infusion 1996(May); 13-26.

14. Dracup K, Walden JA, Stevenson LW, Brecht M-L. Quality of life in patients with advanced heart failure. J Heart Lung Transplant 1992; 11: 273 79

15. Grady KL, Jalowiec A, White-Williams $C$; et al. Predictors of quality of life in patients with advanced heart failure awaiting transplantation. J Heart Lung Transplant 1995; 14: 2-10.

16. Forgosh L. Outpatient inotropic infusions and the impact on quality of life in patients with advanced heart failure. Cardiovascular Reviews \& Reports 1997; 18: 11-22.

17. Heaney L, Antonio C. Outpatient infusion clinics: a strategy for the successful management of advanced heart failure. Journal of Cardiovascular Management 1997(Jan/Feb); 12-16.

18. Marius-Nunez AL, Heaney L, Fernandez RN, et al. Intermittent inotropic therapy in an outpatient setting: a cost-effective therapeutic modality in patients with refractory heart failure. Am Heart J 1996; 132: 805-08. 